\title{
Qos Parameters Comparison for 802.11 B, A\&G Standards
}

\author{
Arun Uniyal ${ }^{1}$, Rashmi Chaudhary $^{2}$ \\ ${ }^{1}$ (Electronics and communication,D.I.T.,Dehradun/Uttarakhand Technical University,India) \\ ${ }^{2}$ (Electronics and communication,D.I.T.,Dehradun/Uttarakhand Technical University,India)
}

\begin{abstract}
Wireless networking refers to the technology that enables two or more nodes to communicate over radio frequency, using a network protocol. Advancement in wireless networking technology, wireless local area networks (WLANs) are used almost everywhere like Home, Business and in corporate environments etc. In this paper theoretical analysis has been used to predict the Bandwidth efficiency, total packet delay, protection mechanisms and power consumption in IEEE 802.11a, 802.11 b and 802.11g WLANs .
\end{abstract}

Keywords: IEEE 802.11b, IEEE 802.11a, IEEE $802.11 \mathrm{~g}$

\section{Introduction}

The use of wired Local Area Networks has become ever more common place, even in situations where only a few computers need to be connected together. Advantages like cost reduction, High robustness and easy system configuration making WLAN more popular. Three different PHY layers are available for the IEEE 802.11 WLAN as shown in Table 1.1 [1][2][3]. IEEE 802.11b radios transmit at $2.4 \mathrm{GHz}$ and send data up to 11 Mbps using direct sequence spread spectrum (DSSS), infrared (IR), and frequency hopping (FH) [2]; whereas IEEE 802.11a radios transmit at $5 \mathrm{GHz}$ and send data up to 54 Mbps using orthogonal frequency division multiplexing (OFDM) [1]. The IEEE 802.11g standard [3], extends the data rate of the IEEE $802.11 \mathrm{~b}$ to 54 Mbps in an upgraded PHY layer named extended rate PHY layer (ERP).The big benefit from Wireless LANs is increased mobility. The IEEE designed 802.11 to support medium-range, higher data rate applications, such as Ethernet networks, and to address mobile and portable stations.802.11 is the original WLAN standard, designed for 1 Mbps to 2 Mbps wireless transmissions.

\begin{tabular}{c|ccc}
\hline Characteristic & $802.11 a$ & $802.11 b$ & $802.11 \mathrm{~g}$ \\
\hline Frequency & $5 \mathrm{GHz}$ & $2.4 \mathrm{GHz}$ & $2.4 \mathrm{GHz}$ \\
Rate (Mbps) & $6,9,12,18,24,36,48,54$ & $1,2,5.5,11$ & $1,2,5.5,6,9,11,12,18,22,24,33,36,48,54$ \\
Modulation & BPSK, QPSK, 16 QAM, 64 QAM & DBPSK, DQPSK, CCK & BPSK, DBPSK, QPSK, DQPSK, CCK \\
& (OFDM) & (DSSS, IR, and FH) & 16 QAM, 64 QAM (OFDM and DSSS) \\
FEC Rate & $1 / 2,2 / 3,3 / 4$ & $\mathrm{NA}$ & $1 / 2,2 / 3,3 / 4$ \\
Basic Rate & $6 \mathrm{Mbps}$ & 1 or 2 Mbps & 1,2, or 6 Mbps \\
\hline
\end{tabular}

Table 1 Characteristics of various PHY layers in the IEEE 802.11 standard

The evolvement of WLAN 802.11 give birth to some more extended standards like IEEE $802.11 \mathrm{~b} / \mathrm{a} / \mathrm{g}$.The IEEE $802.11 \mathrm{~b}[4]$ standard provides data rate upto $11 \mathrm{Mbps}$ at a frequency of $2.4 \mathrm{GHz}$. While the IEEE 802.11 a standard enhances the data rate upto $54 \mathrm{Mbps}$ but it has not the backward compatibility with IEEE $802.11 \mathrm{~b}$.The IEEE $802.11 \mathrm{~g}$ standard is the combination of both previous standards[5] as it has the properties of both the standards. This provides the data rate of 802.11 a at $2.4 \mathrm{GHz}$ and therefore it provides the backward compatibility with IEEE 802.11 and $802.11 \mathrm{~b}$.

\section{Delay and Bandwidth efficiency}

The time travel by a packet to travel from one node to other.It can also be defined as aggregate of backoff time,inter frame spaces and transmission time of all control frames. Unit of delay is typically in micro seconds.

Delay per MSDU(CSMA/CA cycle $)=(\mathrm{T}$ data $+\mathrm{T}$ sifs $+\mathrm{T}$ Ack $+\mathrm{T}$ difs $+\mathrm{T}$ bo $)$

Delay per MSDU(RTS/CTS cycle $)=(\mathrm{T}$ rts $+\mathrm{T}$ sifs $+\mathrm{T}$ cts $+\mathrm{Tsifs}+\mathrm{T}$ data $+\mathrm{T}$ sifs $+\mathrm{T}$ ack $+\mathrm{T}$ difs $+\mathrm{T}$ bo $)$

$\mathrm{T}$ bo $=(\mathrm{CW} \min * \mathrm{~T}$ slot $) / 2$

$\mathrm{T}$ difs $=2 * \mathrm{~T}$ slot $+\mathrm{T}$ sifs

For CSMA/CA ,T data is Transmission time(payload) in $\mu$ s, $\mathrm{T}$ sifs is time(SIFS )in $\mu$ s, T ack is time (acknowledgement)in $\mu \mathrm{s}, \mathrm{T}$ difs is time (DIFS)in $\mu \mathrm{s}, \mathrm{T}$ bo is time(backoff) in $\mu \mathrm{s}$. 
For RTS/CTS, T rts is transmission time(RTS) in $\mu$ s, T sifs is time(SIFS )in $\mu$ s,T cts is transmission time(CTS)in $\mathrm{T}$ sifs is time(SIFS )in $\mu$ s, $\mathrm{T}$ data is Transmission time (Payload), $\mathrm{T}$ ack is transmission time (ACK)in T sifs is time(SIFS )in $\mu$ s, T difs is time(DIFS)in $\mu$ s, $\mathrm{T}$ bo is time

(backoff) in $\mu$ s.T slot is time(slot)in $\mu$ s.

\section{Bandwidth efficiency}

Bandwidth efficiency is also known as spectral efficiency and is defined as the ratio of maximum MAC throughput to the channel bandwidth.

$$
\eta=M M T M A C / B W
$$

\section{Protection Mechanisms:}

To avoid the collision due to OFDM/DSSS interoparabiilty problems and to transmitted data packets of OFDM two different mechanisms are used namely CTS to Self and RTS/CTS respectively. These two mechanisms are defined by the figure 2 and figure 3

\section{RTS/CTS V/S CTS-to-Self Mechanism}

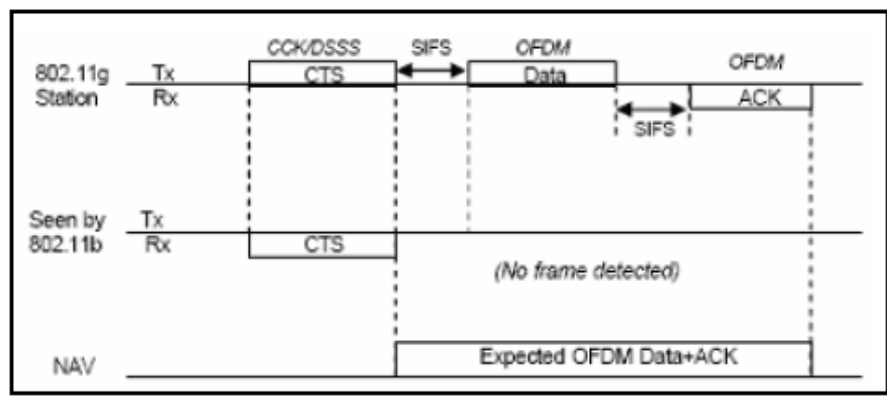

Fig1:CTS to Self mechanism

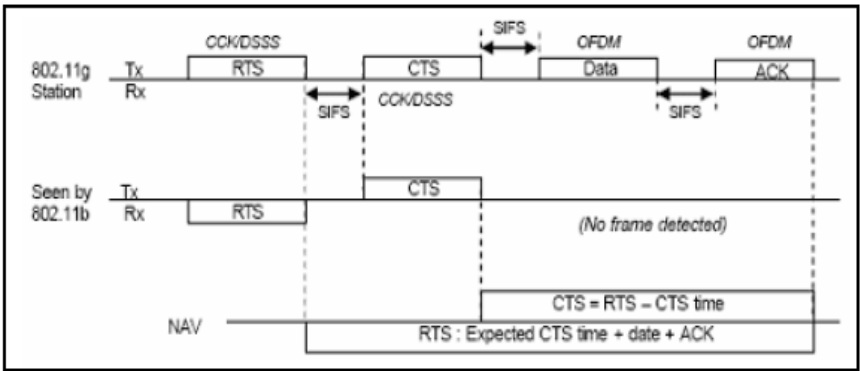

Fig2:RTS/CTS mechanism

CTS TO SELF protection mechanism used in $802.11 \mathrm{~b} / \mathrm{g}$ mixed mode .Whereas $802.11 \mathrm{~b}$ have not ability to receive and decode the OFDM frames. The IEEE $802.11 \mathrm{~g}$ standard defines the CTS-to-self protection mechanism as an alternative to RTS/CTS in order to reduce the overhead packet data added in a WLAN system.CTS-to-self protection mechanism cannot face the hidden terminal problem.

RTS/CTS (Request to Send / Clear to Send) is the protection mechanism used by the 802.11 wireless networking protocol to decrease frame collisions introduced by the hidden node problem. This protection mechanism has more robustness against hidden terminal problem.

\section{Power Consumption}

For new Wi-Fi-enabled devices,power consumption and battery life are the two main critical factors specially for mobiles and PDAs. Around 90 percent of the time is spend in a standby mode foe various WIFI applications as compared to actual data transmitting or receiving. So for low power consumption in stand by mode is a requirement for long battery life.

Other important factor in the overall power consumption of a WLAN device is how long the device must remain in an active mode to transreceive some data.

Theoraticaly $802.11 \mathrm{~b}$ has lower throughput so it consumes less battery power than $802.11 \mathrm{a}$ and $802.11 \mathrm{~g}$.For the similar type of data $802.11 \mathrm{~b}$ consumes around thirty percent less power than equivalent $802.11 \mathrm{a} / \mathrm{g}$ devices.

\section{RESULTS}

The Total Packet Delay is the total delay time from the start of transmission of a packet till it is received by the receiver. The Queuing Delay is expressed as the time a packet waits in a queue till its execution 
starts. The sum of Media Access delay and Queuing Delay is also called Total packet delay.If the packets reaches the router node faster than the process time taken by it.The data is temporarily stored in the buffer. As the buffer size alters maximum queuing delay also changes.

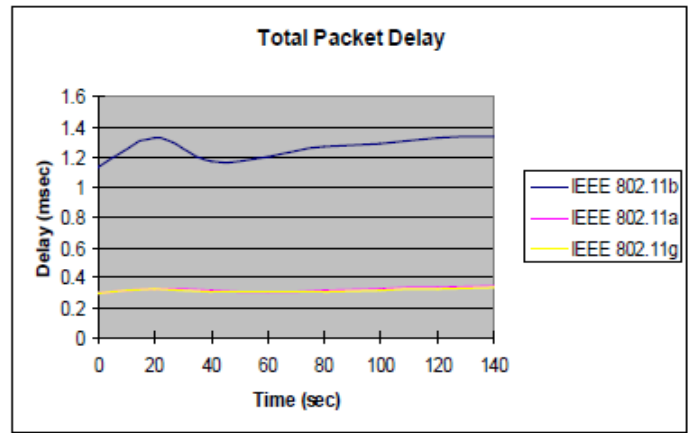

FIG 3:Graph for total packet delay

Above results shows that IEEE $802.11 \mathrm{~b}$ has higher total packet delay as compared to $802.11 \mathrm{a} / \mathrm{g}$.

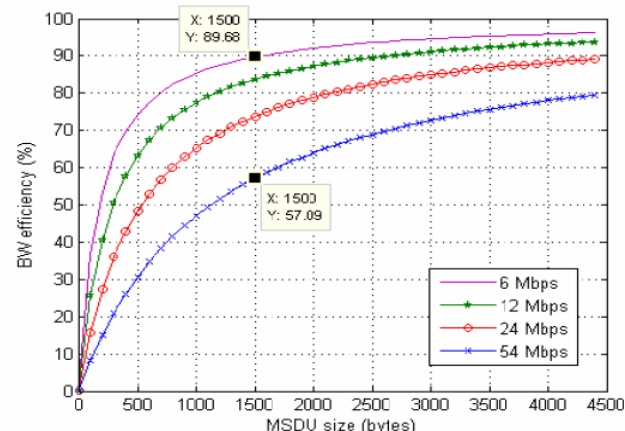

Fig:4 B.W.efficiency of 802.11a

The B.W.efficiency \% curves for different data rates are shown in fig4 with different coloring curves has been plotted.The figure shows that the B.W.efficiency for 802.11a for $6 \mathrm{Mbps}$ is more as compared to other data rates.

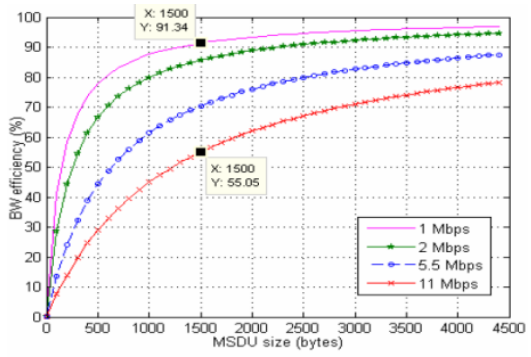

Fig 5:B.W.efficiency of $802.11 \mathrm{~b}$

The graph shows also different curves for various data rates for $802.11 \mathrm{~b}(1,2,5.5,11 \mathrm{Mbps})$. The low data rate like 1 Mbps have greater B.W.efficiency than high data rates like 2,5.5,11 Mbps.

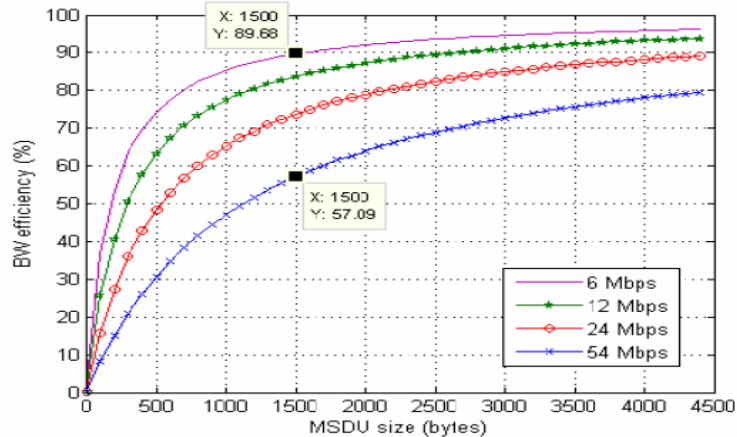

Fig 6:B.W.efficiency of $802.11 \mathrm{~g}$

For $802.11 \mathrm{~g}$ the low data rate have greater Bandwidth efficiency than higher data rate. 
For example for data rate of $6 \mathrm{Mbps}$,for MSDU size 1500 bytes the bandwidth efficiency is 90 .Similarly for data rate 54 Mbps, the B.W.efficiency is lower(60) for 1500 bytes MSDU size.

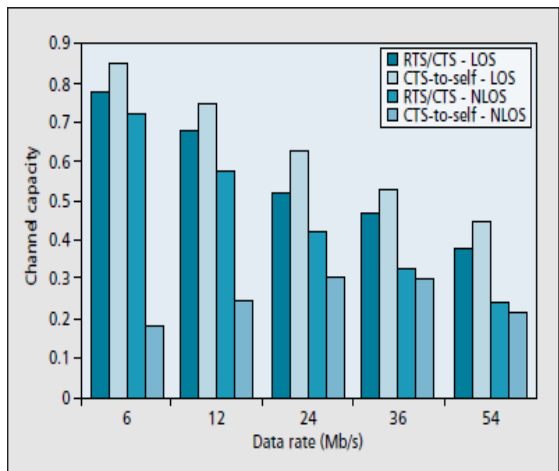

Fig 7:RTS/CTS v/s CTS-to-Self mechanism

When all the stations are in line of site,the efficiency of CTS-to-Self mechanism is more than RTS/CTS mechanism. From the graph shown in fig., for a data rate of $36 \mathrm{mbps}$, the channel capacity for CTS-to-Self mechanism is 0.5 , higher than that when RTS/CTS mechanism is used (0.45).As a result of this the throughput also increases.

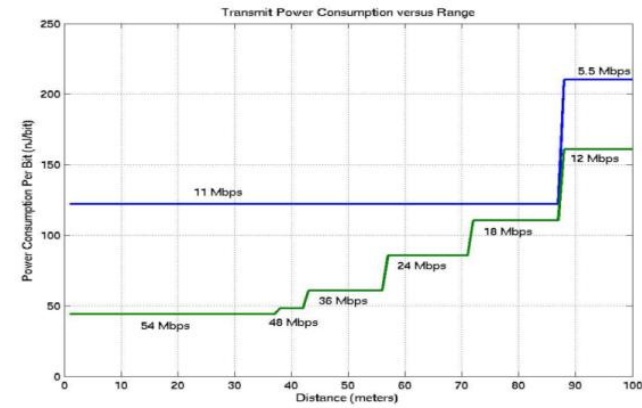

Fig 8: Power consumed per bit v/s dist.curves

The graph shows the comparison in power consumption for $802.11 \mathrm{a} / \mathrm{g}$ and $802.11 \mathrm{~b}$. The graph shown in fig 8 shows the coverage of consumer from the access point node,the rate around various distances from the access point node.

Blue line indicates $802.11 \mathrm{~b}$ and green line indicates $802.11 \mathrm{a} / \mathrm{g}$.

\section{Conclusion}

The results showed that the total packet delay is higher for IEEE $802.11 \mathrm{~b}$ compared to IEEE $802.11 \mathrm{a} / \mathrm{g}$ standards, the CTS-to-self mechanism is more efficient in clear LOS condition but robustness is much lower than the RTS/CTS protection mechanism against hidden terminal problem,also the bandwidth efficiency of $802.11 \mathrm{~b}$ is much greater than $802.11 \mathrm{a}$ and $802.11 \mathrm{~g}$.

The final result shows that the $802.11 \mathrm{a} / \mathrm{g}$ have greater power efficiency than $802.11 \mathrm{~b}$.

\section{References}

[1] IEEE 802.11 WG part 11a, "Wireless LAN Medium Access Control (MAC) and Physical Layer (PHY) specifications, High-speed Physical Layer in the $5 \mathrm{GHz}$ Band," 1999

[2] IEEE 802.11 WG part 11b, "Wireless LAN Medium Access Control (MAC) and Physical Layer (PHY) specifications, Higher Speed PHY Layer Extension in the $2.4 \mathrm{GHz}$ Band,"1999

[3] IEEE 802.11 WG part 11g, "Wireless LAN Medium Access Control (MAC) and Physical Layer (PHY) specifications, Further Higher Speed Physical Layer Extension in the 2.4 GHz Band," 2003.

[4] IEEE Std. 802.11b, "Higher-Speed Physical Layer (PHY) Extension in the $2.4 \mathrm{GHz}$ Band," 2001.

[5] IEEE Std. 802.11g, "Further Higher-Speed Physical Layer Extension in the 2.4 GHz Band," 2003. 